\title{
A review on hybrid optimization algorithms to coalesce computational morphogenesis with interactive energy consumption forecasting
}

\author{
To cite : \\ Lazaros MAVROMATIDIS, A review on hybrid optimization algorithms to coalesce \\ computational morphogenesis with interactive energy consumption forecasting, Energy and \\ Buildings, Elsevier ( IF : 5.879, SNIP : 2.129, SJR : 1.737 ), pages 192-202, Volume 106, 2015, \\ doi:10.1016/j.enbuild.2015.07.003. \\ Lazaros Elias Mavromatidis ${ }^{1^{*}}$ \\ ${ }^{1}$ ICube UMR 7357, Université de Strasbourg, INSA de Strasbourg, 24 Boulevard de la Victoire, \\ 67084, Strasbourg Cedex, France \\ *Corresponding author : lazaros.mavromatidis@insa-strasbourg.fr ; lazarosmavr@hotmail.com
}

\begin{abstract}
The present review paper focuses on the exploration and qualitative evaluation of hybrid optimization methods applied to architectural design, computational morphogenesis and energy consumption problems. After introducing the computational morphogenesis notion and the novel institutional framework of nZEB labeling, we define here computational morphogenesis as a design procedure where the environmental qualities of the envelope and especially thermal storage and load shifting have the potential to guide an automation process of shape creation in the building scale. For this reason we focus on reviewing the well-cited literature on scale that introduced novel hybrid optimization tools especially developed for thermal load, energy consumption optimization and/or computational morphogenesis optimization issues. Different approaches and methods are reported in this review paper, while at the end of the paper an exhaustive list of conclusions and potential perspectives of these approaches is explicitly
\end{abstract}


presented. Inexorably, we seek to review here hybrid optimization tools that are (or could be) applied on computational morphogenesis problems with the aim to optimize, facilitate and encourage a creative architectural design in relation to innovative envelope conception to promote interdisciplinary research coupling the fields of architectural design and building physics.

Keywords : computational morphogenesis, hybrid optimization methods, genetic algorithms, thermal storage, load shifting

\section{Introduction and general context}

In a context where the building sector is responsible for $42 \%$ of final energy consumption, $35 \%$ of greenhouse gas emissions and 50\% of material resources consumption, the European Union introduced a new label to characterize the energy performance of buildings [1], [2], [3], [4], [5]. More precisely, in the general framework of climate change, the re-cast Energy Performance of Buildings Directive (EPBD) requires that from 2019 onwards "all the new buildings occupied and owned by public authorities are nearly zero-energy buildings" (nZEB) and by the end of 2020 "all new buildings are nearly zero-energy buildings" [6], [7], [8], [9], [10], [11]. In other words, this European Directive on energy efficiency of buildings (EPBD) requires member states to ensure that on $1^{\text {st }}$ January 2021, all new buildings have an energy "almost zero" consumption in order to obtain the nZEB label [12], [13]. The aim of this directive is to enhance interdisciplinary research on building applications sharing knowledge about products, systems, conceptual methods and innovative techniques that combine low energy consumption, renewable 
energy and industrialization [14], [15], [16], [17], [18], [19], [20], [21]. Hence, as we understand, the first step, to achieve nZEB levels of energy consumption consists in designing buildings with high thermal storage and load shifting potential. And at this stage we understand that we have to enter in the equation the building form optimization at early design stages in order to achieve high standards of sustainability.

When we deal with a classic building design problem we work simultaneously during two distinct phases : the shape conception and the ex nihilo post conceptual phase where the geometry of the problem is defined in detail and engineering accomplishes the tasks of constructability and sustainability. In general we remark that the shape conception stage remains an integral artistic procedure while energy performance issues are not entered in the equation of form creation. Even if the building research community has been always using building performance simulation tools ([22], [23], [24], [25], [26], [27], [28], [29]), this kind of research is not coupled with the architectural design process at early design stages. As it is well-argued elsewhere, there are many reasons for this delay, starting from the difficulty of using complex sophisticated simulation tools to describe complex physical phenomena ([28], [26], [30], [31], [32]), the augmented calculation time needed for such operations, the uncertainty in the results and the general impression that the designer is restricted by the limitations of the tools ([33], [34]). Architectural discourse has always included a reflection on providing a high quality and comfortable interior environment via an equilibrated architectural project, albeit the use of computer-aided building energy simulation software in the decision-making process for building form optimisation at early conceptual stages is limited. In architectural design, regarding the sustainability of the created space, intuition played the most important role, and the fact that 
traditional architecture has always sought to use local materials and adapt building forms, systems and space organization to precise climatic conditions optimizing their thermal storage capacity, reinforced a kind of discourse among architects and designers, which generally concludes that the use of thermal simulation tools at an early design stage is inessential.

However, the use of procedural, parametric and generative computer-supported techniques in combination with mass customization and automated fabrication is not new in architectural design and consists an alternative way for building form conception. Such tools are the main numerical procedures currently used in contemporary architecture to enable holistic manipulation and the subsequent production of increasingly complex architectural arrangements. In this paper we will try to link the currently employed generative computer-supported techniques and concepts with the sustainable dimension of architecture in order to propose the main elements to reinvent computational morphogenesis in the climate change era.

\subsection{Definition of computational morphogenesis in architecture}

In recent architectural discourses, the approaches to conceive and design original architectural forms from scratch have been described as morphogenesis or more precisely computational or digital morphogenesis. By definition, computational morphogenesis is a process of shape development enabled by computation. Although the concept is applicable in many scientific fields, the term computational morphogenesis was first used in architecture and engineering. Ergo, in architectural design, computational morphogenesis is a group of methods that employ digital media and numerical techniques to shape the form and adapt it in a specific context rather 


\section{Dr Lazaros MAVROMATIDIS, PhD \& HDR Architect-Engineer}

than simply expressing an individual's inspiration offering an original representation. According to Roudavski, in this inclusive perspective, computational morphogenesis in architecture bears a largely analogous or metaphorical relationship with morphogenetic processes in nature, expressing the dependence of progressive development; nevertheless it not necessarily refers to the adaptation of the current mechanisms of growth [35]. Meanwhile, recent speeches have linked computational morphogenesis in architecture at a number of concepts such as emergence, self-organization and sustainable or ecological design. According to Roudavski [35]:

«A better understanding of biological morphogenesis can usefully inform architectural designing because : 1) architectural designing aims to resolve challenges that have often already been resolved by nature; 2) architectural designing increasingly seeks to incorporate concepts and techniques, such as growth or adaptation, that have parallels in nature; 3) architecture and biology share a common language because both attempt to model growth and adaptation (or morphogenesis) in silico. » ([35], p. 348)

\subsection{Aims and scopes of the present review article}

Auxiliary, since architectural production is nowadays commercialized and industrialized in a general context where climate change consists the major challenge of our era, the research for innovative architectural forms that reach nZEB levels of energy consumption aims to couple optimization techniques with computational morphogenesis. That means that an optimized shape conception could significantly improve thermal storage and load shifting in a given climatic and topographic context. Henceforth, the first question that arises when we deal with a space creation 
problem is the volumetric characteristics and the materiality of the form. That means that when the architect conceives a building form he deals indirectly with the creation of a building envelope. Every form is conceived on the basis of material choices while the thermophysical properties of these materials will play decisive role on the thermal behavior of the form as well as on thermal storage and load shifting of the future building. Thermal engineering research showed that safe sophisticated no toxic materials, with low energy content and low environmental impact is a very important question that should be investigated at an early design stage, since optimum utilization of such materials could significantly influence on the geometry of the envelope [36], [37], [38], [39], [40], [41], [27], [42].

Besides, numerical thermal engineering studies in building scale showed that the control of building forms at an early design stage could eventually lead to the use of local materials (wood, stone, metal...) and to the building form optimization through the regeneration of a contemporary iconic architecture born from the necessity of the reduction of the environmental impact of buildings [43], [44], [45], [46], [47], [48]. However, in the majority of cases, instead of focusing on the above mentioned questions regarding the environmental impact of his conception, the conceptual model of the architect is often based on a simple synthesis of geometrical schemes allowing her/him to move quickly towards a definite geometry that illustrates the overall shape of a building including her/his program's constraints via a simplified sketch and ignoring at the same time to focus on the environmental question. Then thermal engineering accomplishes a posteriori the task of sustainability, trying to provide the best 


\section{Dr Lazaros MAVROMATIDIS, PhD \& HDR Architect-Engineer}

material solution in a given geometrical context through energy consumption calculation under dynamic conditions.

However, since the nZEB label is introduced, the nowadays discourse on the shape of the building with all its components, geometrical, topological, functional, structural, aesthetical, energy efficient, and environmental, is at the center of contemporary scientific and architectural interdisciplinary discourse especially in Europe [49], [50], [51]. In this framework the development of original optimization techniques mainly adapted to computational architectural morphogenesis with the aim to improve thermal storage and load shifting of the conceived building enveloppes became an attractive research field worldwide. Digital representations of architecture have been for several years a creative element for design research. However, this paper aims to review findings in the field of performance-driven architecture, describing hybrid optimization algorithms developed especially for eco-performance and creative architectural design optimization. Hence, our main objective is to review here existing hybrid optimization techniques that can be developed with the objective of reducing the gap between architectural design and current scientific knowledge to optimize the building form through computational morphogenesis in early design stages, reducing the overall energy consumption.

More precisely, the present review paper aims to investigate the question of existing hybrid evolutionary mechanisms developed for energy consumption optimization that can be easily adapted to architectural morphogenesis towards the objective to improve thermal storage and load shifting reaching nZEB levels of energy consumption. Past litterature on computational morphogenesis reveals that bio-inspired design methods played a very important role in the 
recent history of contemporary architecture, eventually describing the biological inspiration not as a formal or organizational analogy but rather as a procedural analogy. Besides, evolutionary design nomenclature in the field of architectural morphogenesis distinguishes evolutionary optimization and evolutionary creativity without linking creativity to sustainability. Ignoring the question of energy performance, different families of optimization algorithms associated with evolutionary design have been developed especially for computational morphogenesis contexts, while simultaneously these algorithms are divided into different categories currently used for pure building physics optimization problems: genetic algorithms, genetic programming, evolutionary strategies, evolutionary programming, etc.

Howbeit, rigourous optimization studies that focus on environmental and energy issues and building form optimization coupled with a variety of different optimisation methods are very limited. Momentarily, after replacing the passive solar conception in the history of architecture, exemplified by some characteristic achievements, the principles of contemporary passive solar conception have been modified because of the new institutional framework imposed by the introduction of nZEB labelization. Wherefore, in the chapters that follow we will briefly present the particularity of design optimization problems and review available hybrid optimization methods that had already been employed for building energy performance issues and have the potential to be adapted in computational morphogenesis optimization problems. Additionally we will also describe the different calculation assumptions behind these algorithms for overall energy consumption estimation in terms of heat transfer, thermal storage, load shifting and daylight availability. Finally, we will provide a list of the potential inputs and outputs in a typical 
complex computational morphogenesis problem coupled to real time energy consumption forecasting.

\section{General design optimization problem statement and solution process}

Since the potential for delivering a variety of optimized building shape's in less time, compared to manual space creation, makes automated design optimization a very attractive alternative, recently, architects are rapidly adopting automated design optimization technology for digital morphogenesis purposes [35]. However, in comparison to other scientific disciplines, in architectural design, the characteristics of the design space are typically unknown until the entire design space has been explored [35]. That means that we have to deal with a permanent dynamic optimization problem. Beyond the bounds, the design space plays a very important role not only in the numerical thermal performance evaluation, but also in the selection of the appropriate optimization search algorithm since computationnal time is very important, while the primary role of this algorithm is to investigate in a robust way the characteristics of the design space. Hence, we understand that we have to deal with a typical "chicken and egg" problem.

Besides, selecting the best method to use and then manually or automatically tuning its parameters is a time-consuming process, largely based on trial and error. Therefore, architectural conception is a real time investigation that doesn't offer the possibility to the architect to solve the same optimization problem multiple times in order to identify the best method or settings that yield the optimal solution or a variety of potential solutions. In adition, the complexity of a typical architectural problem and especially the interactive thermal investigation of the 
conceived space at an early design stage, where thermophysical properties are needed to estimate enveloppe's thermal inertia (an element that is needed in order to constantly feed the optimization algorithm with inputs) makes design evaluations to be computationally expensive and many times we have to perform CPU programming to reduce the calculation time.

As an answer to all the abovementionned specifications, the application of hybrid algorithms that have been developed to rapidly perform in a satisfactory way over a wide range of problems eliminating manual tuning and yielding the desired solution without consuming enormous computational time, significantly reducing manual effort and design time in computational morphogenesis and building thermal performance issues is discussed in the present review paper.

Withal, since to obtain an accurate evaluation employing these algorithms requires several hours or even several days to complete, and having in mind that in a typical architectural design problem a huge number of evaluations are needed to identify an optimized solution, especially when we insert the parameter of nZEB labelisation, then complex CPU programming may be required. As it is revealed in global litterature, the algorithm that best fits to such a problem consists in reducing the total computational time to perform the number of evaluations needed to find one or a variety of optimized solutions (depends on the problem definition and our needs). In this case the selection of the most adequate algorithm has a large impact on the optimization results via the computational time, since the difference in performance between two algorithms can be translated into hours, days or even weeks even if sophisticated CPU programming is performed. 
Generally the typical optimization problem statement is translated in mathematical terms as follows :

Minimize $f\left(x_{1}, x_{2}, \ldots, x_{n}\right)$

such that $g_{i}\left(x_{1}, x_{2}, \ldots, x_{n}\right) \leq 0, \quad i=1,2, \ldots, p$

and $h_{j}\left(x_{1}, x_{2}, \ldots, x_{n}\right)=0, \quad i=1,2, \ldots, q$

where :

$f\left(x_{1}, x_{2}, \ldots, x_{n}\right)$ is the objective function,

$g_{i}\left(x_{1}, x_{2}, \ldots, x_{n}\right)$ are the $p$ inequality constraints,

$h_{j}\left(x_{1}, x_{2}, \ldots, x_{n}\right)$ are the $q$ equality constraints,

$\left(x_{1}, x_{2}, \ldots, x_{n}\right)$ are the $n$ design variables.

In typical computational morphogenesis problems in architecture, we consider a problem of the following form:

$\max _{x} f(x)$ subject to $g_{j}(x) \leq c_{j}$ for $j=1, \ldots, m$, where $f$ and $g_{j}$ are functions of $n$ variables, $x=\left(x_{1}, x_{2}, \ldots, x_{n}\right)$ and $c_{j}$ for $j=1, \ldots, m$ are constants. The functions $f, g_{i}$, and $h_{j}$ are responses of the system, while the design variables $\left(x_{1}, x_{2}, \ldots, x_{n}\right)$ are the inputs of the problem.. In general, the responses $f, g_{i}$, and $h_{j}$ are not known analytical functions. The values of these functions can be calculated at a finite number of trivial distinct points, cloud points or sophisticated hybrid morphogenetic designs (depends on the morphogenetic engine selected prior to the algorithm's development) and could refer to the available direct solar radiation, the wind velocity in precise 


\section{Dr Lazaros MAVROMATIDIS, PhD \& HDR Architect-Engineer}

points, overall mean time lag, overall mean decrement factor, mean $U$ factor etc, based on the strategy embedded in every different optimization algorithm. However the level of difficulty of the problem is increased, since the evaluation of each response corresponding to a potential global shape design must be performed using an analysis model (such as a finite element model, a control volume model, CFD models, dynamic simulations, or on the other hand any other simplified predictive model). The role of an optimization algorithm is to solve the abovementionned problem using as few design evaluations as possible in less computational time with the aim to provide -in the measure of possible- interactive evaluations (real time).

So, from this point of view research on building physics to optimize thermal storage and load shifting is developed towards the following two parallel axes:

i) development of original, robust and sufficiently sophisticated optimization algorithms that can browse a huge design space in order to propose the best family of morphological solutions,

ii) development of original, simplified, robust enough and validated thermal analysis models that can provide a real time forecast regarding the environmental impact of the building form through the entire life cycle of the building.

In this review paper we focus in the objective of a typical optimization problem in computational morphogenesis that is to size the various components of the building proposing $3 \mathrm{D}$ geometric forms put in a specific geographical context (in terms of geometry and climate characteristics) in a manner that we obtain not a simple form but an original complex iconic symbol. In the framework of sustainable development and nZEB labelisation the main aim is to optimize 
thermal storage and load shifting, while meeting high ecological performance characteristics for the entire life cycle of the building starting from construction, maintenance and even deconstruction at the end of the building's lifetime (Figure 1).

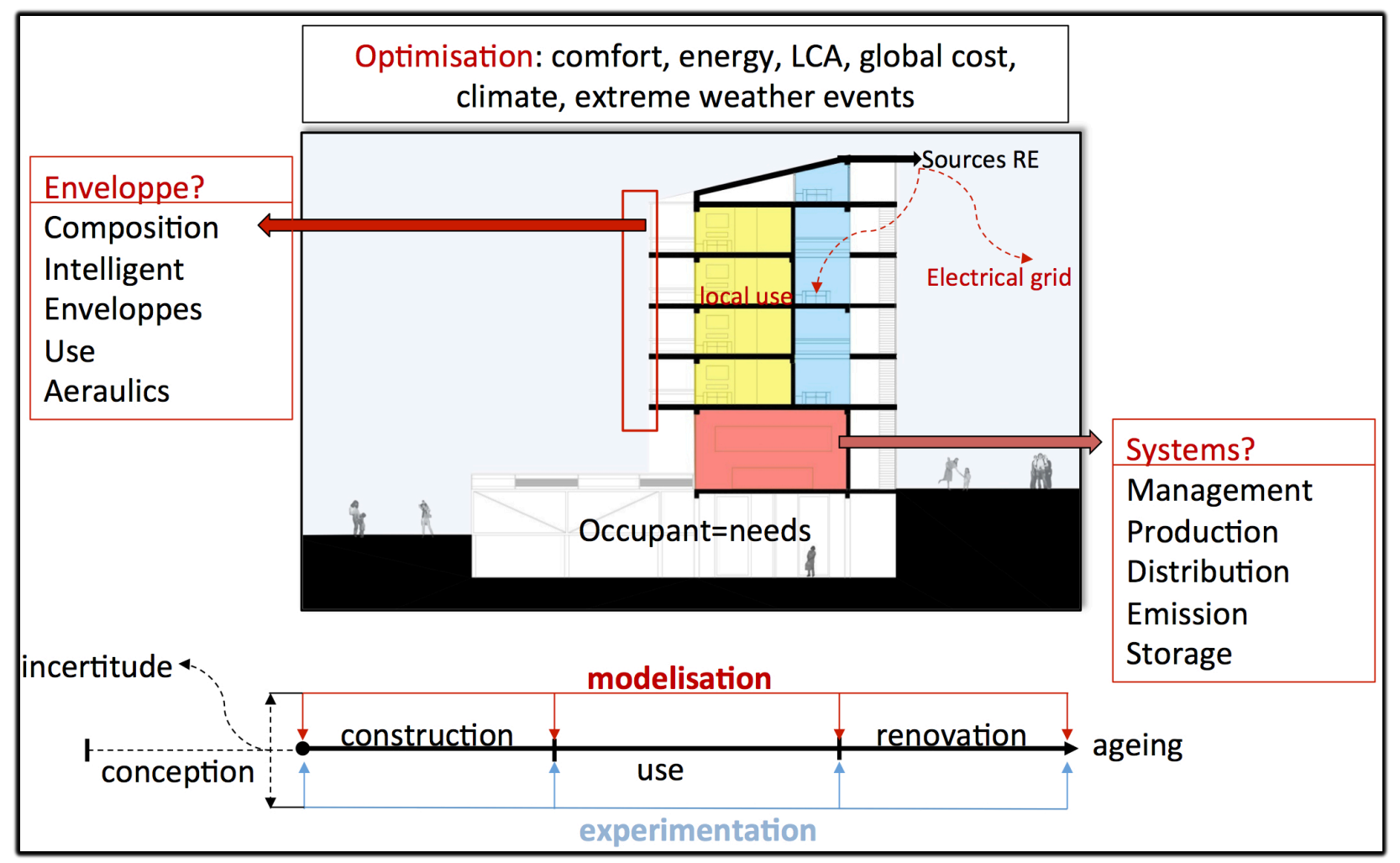

Figure 1: Objectives, input and output parameters of a typical optimization problem in computational morphogenesis throughout the entire lifetime of the building in the institutional framework of the nZEB label.

Since nZEB label is officially introduced the main objective of such optimization problems is not only to minimize building energy consumption, but also to maximize the in situ energy production passing from thermal storage and load shifting optimization. On that account, as we will see in the following chapter, due to the difficult design landscape of the problem a variety of 
optimization algorithms have been employed in the past for such optimization studies.

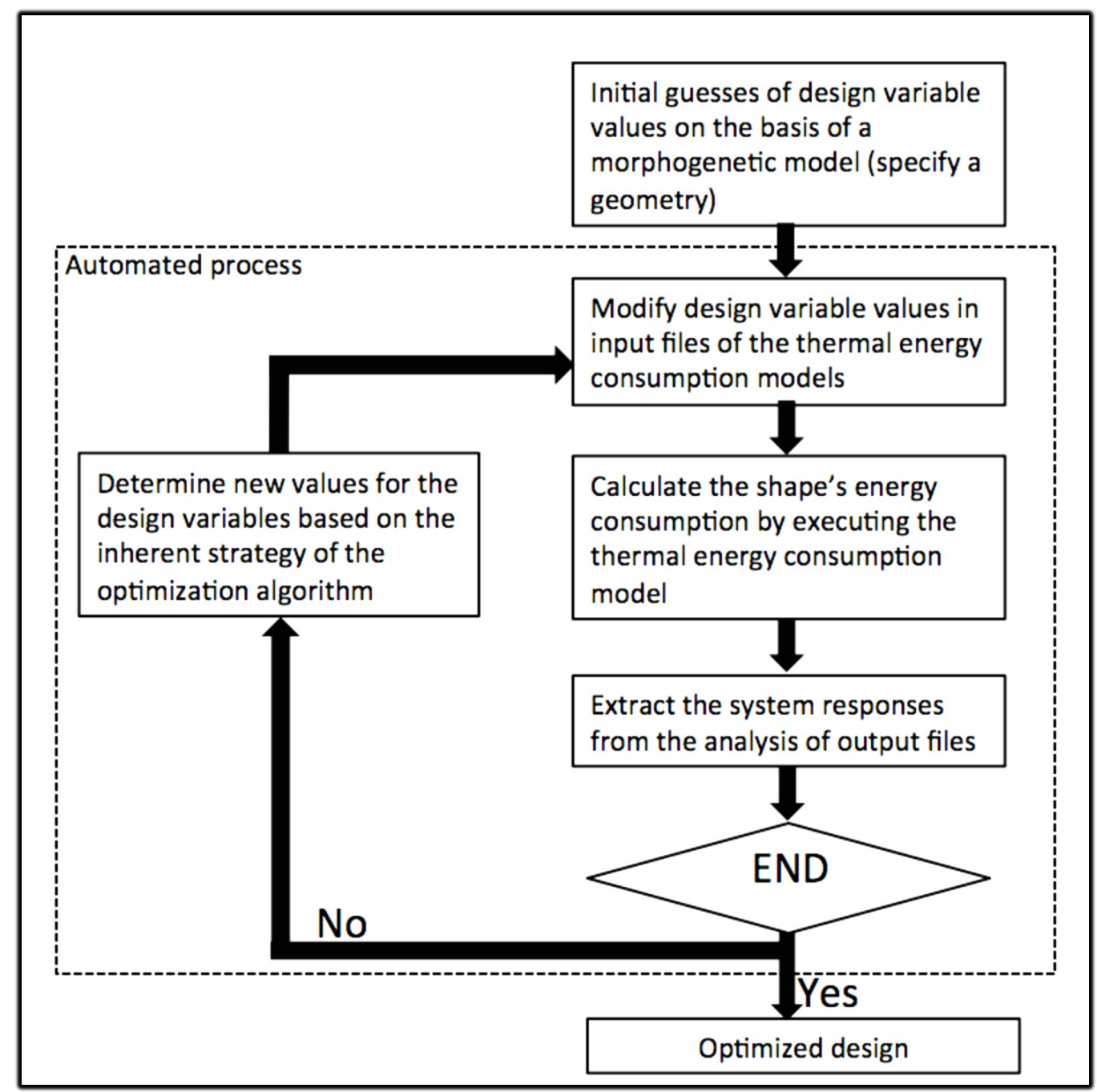

Figure 2: General automated design optimization process flowchart for computational morphogenesis problems. The automation process of the hybrid optimization algorithm may already involve both the first "guess" or seed parameters.

Finally, the solution process of such a complex general design optimization study is illustrated in Figure 2. The main advantage is that intermediate iteration steps within the dashed box occur automatically. In general, prior to execution of the optimization study one or more than one 
thermal heat load model(s) should be created to forecast the shape's overall energy consumption in the given geographic and climatic context of the study. The input file(s) regarding the shape and the materiality of the form are associated with these models. These input files are constantly modified by the optimization tool for each new design evaluation during the study. Hereinafter we understand that both analysis model(s) and optimization algorithm(s) play a very important role in order to obtain accurate results since both of them control the type and direction of our research at each iteration step. Denote that the level of optimization algorithm's sensitivity to the initial guesses (or seed parameters) of design variable values varies. So in order to choose the best type of optimization algorithm for our study, we have to investigate in which degree of precision we will provide the initial boundaries of our study.

\section{Types, categorization and general overview of main optimization algorithms used for computational morphogenesis and building thermal performance optimization}

Optimization algorithms can be classified in the following two big categories:

- Monolithic algorithms: are called those algorithms in which only a single scheme is used. That means that monolithic algorithms typically use a single strategy in order to provide optimum designs, and repeat the same actions at each cycle. Examples of monolithic algorithms used on building energy performance and computational morphogenesis optimization problems, are response surface methods, genetic algorithms, gradient-based hill-climbing schemes or simplex schemes, etc.

- Hybrid algorithms: are those optimization algorithms in which multiple methods or strategies are employed within a cycle, or during each cycle. The goal of a hybrid 
algorithm is to take advantage of the strengths of several strategies during a single optimization study. Past research that focus on computational morphogenesis optimization methods provided us with many interesting hybrid algorithms in which many different methods have been combined to obtain the best optimum solution reducing the computational time. Examples of hybrid algorithms especially developed for building energy performance and computational morphogenesis optimization problems can be found on the literature and are based on web-based parallel genetic algorithms [52], modified simulated annealing algorithms [53], genetic algorithms coupled with artificial neural networks [54] or multi-linear regression meta-models [55], etc.

In addition to the above definitions, algorithms should be also categorized as static or adaptive. This classification is an indirect index of the sensitivity of the optimization algorithm to the input fixed boundaries of the problem. A static algorithm contains tuning inputs that must be set prior to execution of an optimization study, and these parameters remain fixed throughout a given run. For example, in a genetic algorithm, the user must specify the population size, the crossover type and rate, the mutation type, etc. Hence, the performance of a static algorithm on a given problem is a function of the definition of its parameters. Furthermore, an experimented user should tune a static optimization algorithm in order to provide accurate results. This procedure may be time consuming and in order to be achieved with high precision an experts knowledge should be mobilized. On the other hand, an adaptive algorithm automatically adjusts itself during a run based on statistics, heuristics, or other feedback in relation to the problem solution process. Adaptive algorithms eliminate the need for a user to tune the solution process. As a consequence an adaptive algorithm is a more dynamic optimizing technique, which provides better results 
during the first search process if the automated adaptation process works in a robust way.

In the following subchapters we proceed to a brief description of the different optimization methods currently used for building energy consumption and computational morphogenesis problems in architecture.

In recent years it has become evident that a skilled combination of an optimization algorithm with other optimization techniques, the so called hybrid algorithm (or metaheuristic), may provide a more efficient behavior and a higher flexibility. This is because hybrid algorithms have the potential to combine their advantages with the complementary strengths of, for example, more classical optimization techniques such as branch and bound, regression modeling and/or dynamic programming. For this reason, in the recent past, hybrid metaheuristics (such as for example complex genetic algorithms) have been developed to solve a wide range of optimization problems in various fields of engineering and especially in the field of computational morphogenesis. Their potential to solve many hard and extremely complex optimization problems effectively establishing at the same time powerful search capabilities for solving such kind of problems in a reasonable calculation time is in the center of interest of the research work that is reviewed in this paper. On the other hand, in spite of the above-mentioned advantages, these kinds of hybrid optimization methods still encounter challenges when solving complex scale optimization problems, forcing the development of new solution procedures whose efficiency is measured by their ability to find acceptable solutions within a reasonable computational time. 
Since hybrid intelligence is recognized as an integration of different metaheuristic approaches and concepts to overcome individual limitations and achieve synergistic effects, the interest for reviewing such optimization methods is the fruit of the will to create links between computational morphogenesis and thermal energy consumption optimization in a synergistic way. Such hybridizations can be used to take the advantage of strengths from different intelligent techniques of each discipline (building physics and architecture) and overcome the difficulties when we try to solve the problems individually.

Hence, the aim of this review paper and especially of the following sub-chapters is to archive the innovative advancements and mathematical modeling of hybrid intelligence for building physics and computational morphogenesis optimization problems, which may depend largely on methods from computational intelligence and operations research. Furthermore, the present paper has as main objective to review and disseminate quality research on hybrid optimization techniques with emphasis on the application of such techniques in the context of computational morphogenesis and building thermal performance optimization. So the following results section is developed in the four following axes :

- Present in synthetic way available hybrid optimization techniques that focus on the optimization of energy consumption and distribution on defined building geometries

- Explore theoretical and technological advancements in mathematical methods for the development from scratch of hybrid optimization techniques, for computational morphogenesis coupled with interactive energy consumption forecasting/estimation

- Review the potential improvements regarding the application of such hybrid optimization 
techniques for real-world and large-scale optimization architectural problems with the aim to reduce calculation time and achieve high standards of robustness even if the complexity of each singular problem remains in high levels.

- Propose a framework of guidelines regarding the definition and the methodology to deal with computational morphogenesis coupled with interactive energy consumption modeling.

Thus, since space is limited here, the detailed mathematical formulation of the methods mentioned below is left to the references cited for each presented research work.

\subsection{Response Surface Methods}

In this simple approach, the design space is sampled at a number of locations using a LatinHypercube (LHC) sampling scheme. Based on the solution at selected points located in the extremities of the investigated domain, an approximate response surface is independently fit for each objective and constraint, and the resulting analytical surfaces are simultaneously searched to find an optimal solution [56]. As we will see in the next sub-chapter, in building energy consumption optimization problems and/or computational morphogenesis optimization problems response surface methods are systematically coupled to hybrid genetic algorithm schemes in order to accelerate in a synergistic way optimization process and provide more consistent and robust results. The effectiveness of this method depends on having a sufficient number of welllocated sampling points, in order to obtain a critical response surface that accurately represents the entire design domain and constraints. Generally it is an effective method for searching the approximate surfaces [56]. However if this method is used as a monolithic optimization scheme 


\section{Dr Lazaros MAVROMATIDIS, PhD \& HDR Architect-Engineer}

for several computational morphogenesis and thermal consumption optimization problems this method was found to be very inaccurate, yielding results that were generally much poorer than those provided by other methods [57], [58], [25], [59], [26].

\subsection{Monolithic and Hybrid Genetic algorithms (GA) employed on building energy consumption optimization and computational morphogenesis}

The Genetic Algorithms are a multi-point, evolutionary optimization method that performs global exploration of the design space while searching for an optimal solution. In, 1986 Goldberg [60] bring together in an explicit manner the computer techniques, mathematical tools, and research results that help us to understand how to apply genetic algorithms to problems in many fields. The main advantage of this method is that it does not require the calculation of solution gradients and for this reason this optimization technique became very popular among researchers that focus on computational morphogenesis optimization. As we will see, the genetic algorithms is an optimization technique that has been used coupled with other optimization methods such as regression analysis and regression models, hierarchical adaptive network-based fuzzy inference system (HANFIS), etc. So, in order to provide to the reader a clear image on how this coupling could help us to extrapolate very important data and optimize the responses into our domain of investigation, we focus here in a limited selection of several characteristic and well-cited studies that consist our main references regarding the application of such hybrid algorithms on computational morphogenesis and building energy consumption optimization problems. 
Let's start with the work of Siddharth et al. who worked on a pure energy consumption problem [61]. Initially, they identified the building system variables that are critically correlated with the building energy consumption (geometry, materiality, occupancy, etc) and their algorithm provided huge populations on the basis of a large number of combinations of the identified parameters. Then for each of such combinations, Siddharth et al. performed building energy simulations for location-specific weather data to determine the system configuration that would result in the facility's reported annual energy consumption. Their genetic algorithms have been utilized to generate such database. Then employing this database, a nonlinear regression fit between the system variables and the annual energy consumption was formulated. Using the regression fit, system configuration for the target energy efficiency is determined, with corresponding energy conservation measures. Finally they also carried out a cost analysis, to prescribe cost effective energy conservation measures. The competency of their tool has been successfully demonstrated in their paper through selected case studies on three different locations with distinct climate conditions.

Additionnally, research has been made to improve reliability of optimization models. Junghans and Darde [53] published a method for improving the reliability of optimization results of genetic algorithms especially developped for building optimization problems. Their methodology consists in combining the genetic algorithm with a modified simulated annealing to verify the calculation results of the algorithm. They tested the prosed hybrid algorithm on a façade optimization problem in different climates, as well as they verified the robustness of the optimization process by a large number of optimization runs and compared to the results of a classic enumerative search method. Likewise, Asadi et al [54] developped from scratch a multi- 
objective optimization model coupling Genetic Algorithm to an Artificial Neural Network applied to a school building case study. However in order to get the exhaustive search results for the predefined problem 75 days would be required since the Artificial Neural Network was time consuming.

Consequently, many researchers worked on the reduction of computational time of such problems, since their complexity significantly increases the time needed to perform a holistic investigation. For example, Yang et al, [52] developed a web-based distributed high-through computation framework with parallel Genetic Algorithm modeling to reduce the computation time of simulation-based building energy optimization problems.

On the other hand, Li and $\mathrm{Su}$ [62] developed from scratch a novel hybrid Genetic AlgorithmHierarchical Adaptive Network-Based Fuzzy Inference System model in which the GA optimizes the structure and number of fuzzy if-then rules in Hierarchical Adaptive NetworkBased Fuzzy Inference System by finding the best parameter values of a subtractive clustering method. In their model, hierarchical structure helps to arrange low-dimensional rule bases and Adaptive Network-Based Fuzzy Inference System is used to optimize the Fuzzy Inference Systems constructed based on the clustering parameter values generated by the Genetic Algorithm. Then they used their hybrid optimization algorithm to predict a hotel's daily air conditioning consumption, while they confronted their calculated results to results obtained using a Neural Network and concluded that their algorithm is more robust in terms of prediction accuracy. Furthermore, Ooka et al [63] instead of employing neural networks, proposed a new optimal design method based on genetic algorithms for energy systems. The originality in their 
work is that they can simultaneously optimize both equipment capacity and operation planning using genetic algorithms. They started their research by conducting a trial analysis, including two optimization stages for one season. In addition, $\mathrm{Xu}$ and Wang [64] proposed a methodology for parameter optimization of a 3R2C thermal network model of building envelopes (composed of three resistances and two capacitances) coupling frequency domain regression and genetic algorithms (GA). They initially calculated the theoretical frequency characteristics of heat transfer through the building envelope using detailed physical description within the frequency range of concern. Then, the frequency characteristics of their simplified 3R2C model were estimated with random values of individual resistances and capacitances, which constrain to total thermal resistance and capacitance. Once, the errors between the theoretical frequency characteristics and the frequency characteristics of their simplified model were calculated they developed a Genetic Algorithm estimator to optimize the parameters of the simplified model, allowing the frequency responses of the simplified model match the actual heat transfer through building envelope the best, providing real time information.

\subsection{Hybrid optimization models especially developed for computational morphogenesis}

In contrast, regarding hybrid optimization models especially developed for computational morphogenesis, there is a tiny literature, since this scientific domain of research is novel. Jin and Jeong [65] published a serie of free-form building shape optimizations on the basis of an original genetic algorithm developed from scratch in the framework of their study. They coupled the algorithm to an established envelope thermal load prediction model that is used as the objective function. They discovered that the variation of thermal load characteristics caused by changing 
the building shape could be rapidly predicted and optimized in the early design phase by architects using Rhino and Grasshopper software for computational morphogenesis. The originality of their work consists on the use of sophisticated weighting factors that made the shape optimization to reflect local climate characteristics, even in the same climate zone. They also calculated the impact of their Genetic Algorithm-based shape optimization on the enhancement of thermal performance of a free-form building and they discovered that this impact was more significant in low latitude regions than in high latitude regions. However the main argument of their work consists in underlining that in the early design phase of a free-form building, the decision of a designer (or architect) is critical, while the building shape plays a key role in the improvement of the final global thermal performance, thermal storage and load shifting. In addition, Wright et al [66] found that the application of a multi-criterion genetic algorithm in the search for a non-dominated (Pareto) set of solutions to pay-off between energy cost and occupant discomfort and architectural form has a very important potential. Their results indicate that the multi-criterion genetic algorithm is able to identify the pay-off characteristic between daily energy cost and zone thermal discomfort exhibiting fast progress towards the Pareto optimal solutions, being able to find feasible solutions within a very few trail solutions.

Finally, Marsault [68], Marin et al [69] and Mavromatidis et al [32], [67], developed EcCoGen, which is a kind of software that belongs to the family of tools based on interactive generative genetic algorithm optimization. The software generates solutions evaluated according to certain criteria, including mainly energy performance and daylight issues. The energy evaluation included in the software features is performed employing sophisticated regression models. The polynomial models incorporated in the optimization software resulted from simple factorial and 
fractional factorial simulation plans, and are conceived in order to forecast a composite dynamic envelope's thermal performance in terms of thermal resistance and daylight availability through the concept of daylight factor. At a first stage, the polynomial regression models link the thermal performance of a composite wall to the inclination angle, the thermal conductivity and the thickness of each envelope's layer [32]. In addition, the authors aimed to enhance software's applicability including energy reduction due to lighting issues via visual comfort optimization. Based on this objective, they also developed a simple methodology to optimize the daylight potential of an architectural form at an early design stage. For this reason Doehlert and BoxBehnken simulation designs have been developed in order to classify and discover the most influent parameters to study. Hence, if an architect knows the environment type, the centered opening ratios, the distance the high and the length of all possible shadow masks she/he can estimate with accuracy the daylight potential of different possible solutions (for example solutions with different centered opening ratios) [67]. Since the main aim of their work consists to obtain a calculation software that works in an interactive way, to perform real-time calculations in the framework of the development of EcCoGen software they incorporated these energy consumption thermal models to an originally developed multi-objective and interactive genetic algorithm which meets important requirements: diversity, performance and adaptation to an architect's subjective choices in the sketching or design stages. Finally they tested and validated this algorithm in a precise case study that was based on three fitness related to the minimization of energy consumption [68], [69].

\section{Discussion of results: key points and most important aspects on coupling building physics and computational morphogenesis optimization problems}


The formulation and the priorities of the optimization problem that deals with original morphogenesis in relation to nZEB labelisation, thermal heat storage and low shifting plays the most important role and has a very strong influence on the resulting optimal design as previous studies revealed. After reviewing the literature figure 3 groups all the potential input and output parameters needed by a hybrid optimization system coupled to an external analysis model.

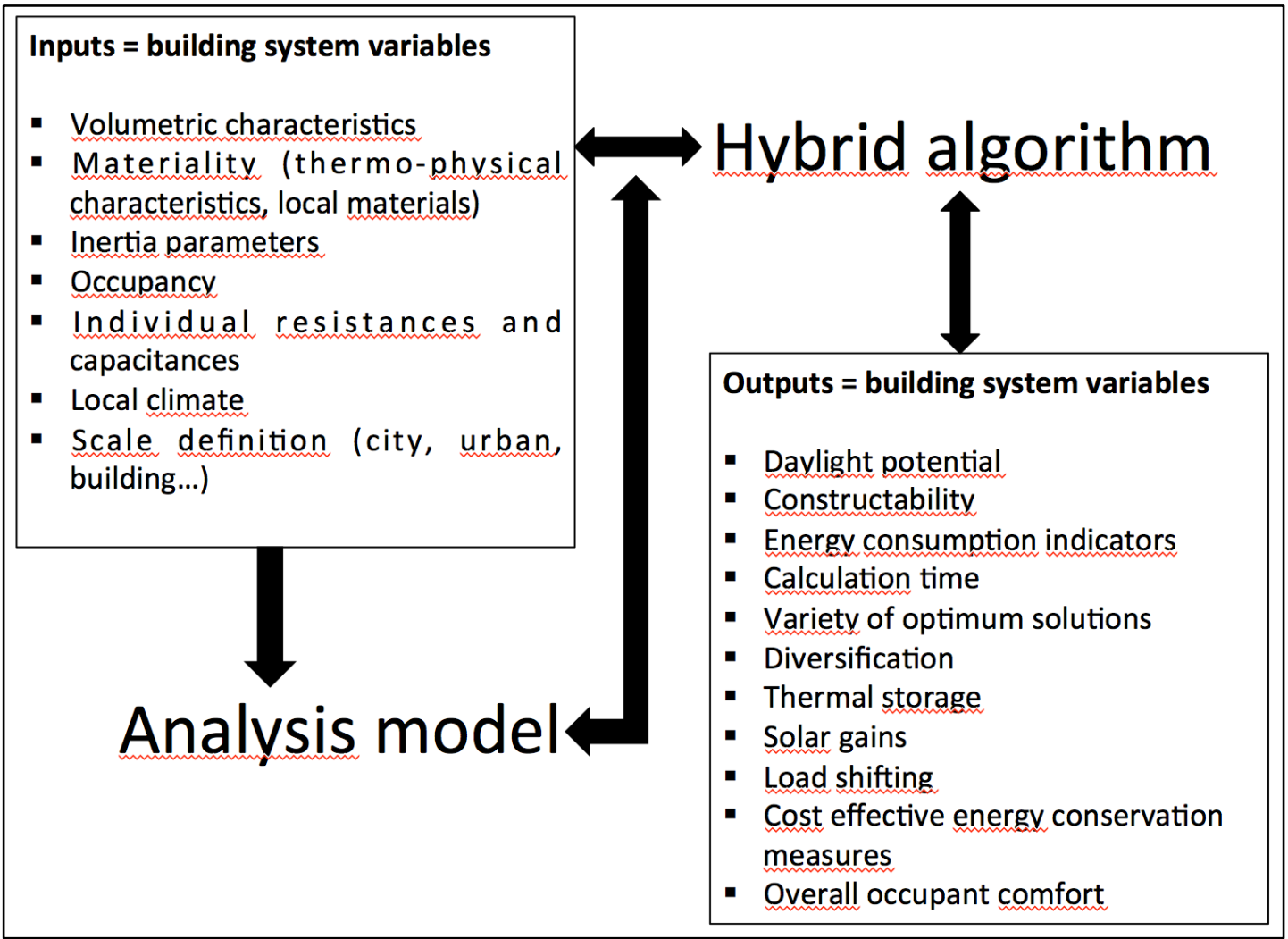

Figure 3: Input and output parameters needed in order to couple a hybrid optimization system coupled and an external analysis model.

Finally, in the present subchapter after reviewing the literature on scale regarding hybrid 
optimization methods applied on building physics we resume here the following key points that have been emphasized in several previous studies and must be considered in every attempt that aims to couple computational morphogenesis with interactive energy consumption optimization. The following synthesis aims to propose a framework of guidelines as follow:

1. Reduce the problem's complexity: creation of simplified energy consumption models coupled with hybrid optimizers

- Many studies typically couple an optimizer with a building energy model, which tends to be time consuming and often leads to suboptimal results because of the mathematical properties of the energy model [57]. To anticipate this, robust simplified energy consumption modeling should/could be performed.

- In order to simplify energy consumption modeling, the alternative to evolutionary algorithms optimization path consists on using polynomial regression models that result from statistical reduction of complex physical modeling on the basis of numerous simulation scenarios that cover the entire domain of the optimization problem. Quadratic polynomial response surfaces constructed using finite element model simulation data and employed as objective functions or design constraints, seem to be a very interesting path that differs from the traditional sensitivity-based optimization, since once the response surface is constructed, there is no need to perform any finite element calculation in each optimization iteration [72]. Furthermore, these kind of meta-models are easily coupled with Non-Uniform Rational Basis Spline (NURBS) modeling that is a mathematical model commonly used in computer graphics for generating and representing curves and surfaces, offering great flexibility and precision for handling both analytic (surfaces 
defined by common mathematical formulae) and modeled shapes [72]. Likewise the Response Surface Methods (RSM) for optimization, may preserve a constant interaction between architects and engineers in an interdisciplinary manner. However, before generalizing such an optimization technique we have to evaluate in detail the response surface method for computational morphogenesis of complex morphogenesis problems where the relation between the design variables and the response quantities of interest is more complex [72].

- The parameters examined through Design of Experiments Methods (or Response Surface Methods) for sensitivity analysis can vary and depend on the problem definition and the aim of the architectural program [22]. Once these parameters have been defined the problem's complexity is significantly reduced and then we should choose the most relevant design factors of the problem in order to perform multi-objective optimization. At this stage in order to reach nZEB performance levels we should focus on many parameters that influence envelope solar potential for energy production, and potential solar gains inserting also in the final equation daylight availability and all these factors should be classified in relation to the given climatic conditions of our problem. For example for hot climates we may have the aim to maximize solar potential for energy production and minimize potential solar gains, while keeping appropriate indoor daylight levels. The employed optimization method can then focus only on the computational morphogenis dimension of the problem and should provide us all the necessary information regarding building regulation and daylight thresholds, which are considered as design constraints, while a variety of potential solutions should be spread on the design space. 
- Studies that had as an objective to develop an optimization path for a free-form computational morphogenesis in relation to the shape's thermal load characteristic at an early design stage focus on Genetic Algorithm techniques since this kind of optimization method can provide a huge variety of potential optimal solutions [65], [75], [43], [76]. Parametric design methods are currently employed for geometric modeling while generally a potential mesh is generated by the $3 \mathrm{D}$ division of the space into finite elements for computational morphogenesis. Then geometric information can be extracted from each finite element and is used to estimate the heat gain and loss characteristics of the whole architectural shape as well as thermal storage and load shifting qualities of the envelope. Past research achieved to couple the above mentioned computational morphogenesis process with genetic algorithm (GA) free-form building shape optimization for a variety of climate zones [65], [66], [77], [32], [67]. The optimization process is based on a variety of established and sufficiently validated-evaluated reduced models that returned the thermal characteristics of free shapes in an interactive way. These simplified models have been used as objective functions, which are the critical required information for a robust GA optimization process, reducing at the same time the complexity of the problem. The results of many researches showed that the coupling of Genetic Algorithms with such simplified established models could rapidly predict and optimize the variation of the heat gain and loss characteristics that was caused by the modification of the overall shape [32], [65], [75], [76], [77], [67].

2. Perform a multiscale optimization: the optimizer should not provide a unique optimum solution but a family of different optimum solutions in order to have the flexibility to adjust the 
final solution in a multi-scale procedure of post-evaluation (from the building to the city scale)

- In thermal performance optimization problems, the design parameters, have significant influence on the final architectural form; so a variety of optimal forms is obtained by changing these design parameters during the project design phase [70]. That means that what is interesting in computational morphogenesis problems, is that we are not looking for THE best solution but for a space of a variety of optimal solutions.

- A building is a part of the whole built environment and to achieve global sustainability, it is necessary to integrate energy and environmental impacts at the highest scope of analysis extending the optimization problem to the urban scale. Furthermore, thermal storage and load shifting are influenced from the topography of each case study. Hence the definition of a holistic indicator for architectural computational morphogenesis is necessary [71].

3. Invent techniques to reduce the overall calculation time to provide interactive optimization overcoming model uncertainties

- To improve building performance stability we need to improve the efficiency of search techniques and approximation methods (surrogate models) for large-scale building optimization problems, quantifying a posteriori the robustness in optimal solutions to significantly reduce computational time [43].

- Even if evolutionary algorithms are a very interesting solution in solving highly constrained envelope, HVAC and renewable optimization problems, allowing to estimate the improvement in the optimality of potential solutions, limitations including model uncertainty, computation time, difficulty of use and steep learning curve exist and so 
future research should work towards the direction to improve such optimization tools [25].

- Simulation based energy consumption optimization problems of complicated building, solved by stochastic algorithms, are generally time-consuming especially when we have to conduct large-scale buildings energy consumption optimizations [52]. However, to anticipate to this reality and reduce the computation time of complex building energy optimization applications web-based parallel GA optimization algorithms based on highthroughput distributed computation environment can be developed [52].

- It has been demonstrated that the Genetic Algorithms do not always provide solutions close to the global optimum and the results of these algorithms can differ significantly from each other because of random search [53]. Nevertheless, evolutionary genetic optimization algorithms (GA) have been massively used for thermal building optimization in the past since they provide a variety of potential optimal solutions even if the optimal solution is close to the global optimum. Hence, in order to improve the robustness of such evolutionary algorithms and assure the optimality of the proposed solutions as well as a higher reliability than what the GA alone can provide by using a relatively short computation time, hybrid single objective building optimization algorithms have been developed to combine evolutionary genetic algorithm schemes with modified simulated annealing algorithms [53].

4. Introduce performance boundaries and couple different optimization techniques to achieve high levels of robustness in less computational time

- Research on multidisciplinary design optimization and performance boundaries for 
design decision supports measured the performance boundaries and concluded that it is the best practice in the context where architects must gauge multiple design concepts with varying complexity coupled with performance objectives through Evolutionary Energy Performance Feedback for Design (EEPFD) [73]. Furthermore it has been underlined that the influence of energy performance feedback on the early design stage is very important information that can significantly influence the energy performance and the environmental impact of the architectural form. Hence the use of performance boundaries for conceptual energy performance design exploration should be explored and evaluated in detail in order to discover the level of retrofit information that should circulate between morphogenetic engines and optimization algorithms at an early design stage. In addition the introduction of performance boundaries reduces the overall calculation time for the study, since the algorithm obtain reference values that serve as the main thresholds that limit the space of the proposed/accepted solutions.

- Alternatively, multi-objective optimization models coupling genetic algorithm (GA) with artificial neural network (ANN) could assist us to quantitatively assess technology choices in a computational morphogenesis retrofit project with the aim to reduce calculation time through synergetic interaction. To reduce the computation time of Genetic Algorithms preserving at the same time their optimization power, past research tried to couple Artificial Neural Networks with Genetic Algorithms [54]. Evidently, this kind of optimization takes place in two steps. Firstly an individual optimization of objective functions focusing on shape's characteristics and performance such as energy consumption, retrofit cost, and thermal discomfort hours is conducted. Then a multiobjective optimization model is developed to study the interaction between the above- 
mentioned conflicting objectives and assess their trade-offs [54].

5. Evaluate the environmental performances of the proposed form in every stage of their life cycle and for a variety of climatic types through intelligent optimizers

- The environmental performances of architectural forms should be evaluated in every stage of their life cycle, since the production phase of used materials and building components can significantly contribute to the total energy consumption and environmental loads. Hence when we deal with computational morphogenesis issues an integrated approach for the optimization of energy and environmental performances of complex geometrical envelopes should be conducted with the aim to combine life cycle assessment, energy simulation and optimization analysis with a variety of techniques [47], [48].

- Since, the thermal overload in several climatic types could be irreversible especially when radiation is not controlled and the free supply of heat indoors is mismanaged the development of different types of multi-objective analysis (for example coupling Genetic Algorithms with Artificial Neural Networks [54]) could become a key element to obtaining several types of high energetic efficiency geometrical forms, through the combination of various materials focusing also on summer cooling loads and optimizing thermal storage and load shifting [74].

\section{Conclusions}

After briefly presenting the newly introduced nZEB label regarding buildings' energy 
consumption, this review paper has considered the main existing hybrid optimization methods found in the literature on scale, which are especially developed for energy consumption optimization in building applications and less for computational morphogenesis in architectural design. The comparison between the different evolutionary optimization methods and the respective modes of computational morphogenesis in selected well-cited case-studies demonstrated that this scientific domain is characterized by complex and flexible mechanisms that can suggest interesting directions for the development of procedural techniques in the architectural domain. Furthermore we can observe that the research domain of computational morphogenesis in relation to thermal storage and load shifting optimization is novel and there are only a few works that explicitly treat this complex optimization problem. This is the main reason that we are interested on this field and the present review paper aims to establish the guidelines for computational morphogenesis coupling with interactive optimization of the environmental impact of the architectural form. Thus, amongst the directions of further future research on this interesting subject, we retain here the following three:

- research on the development of multi-level hierarchies reflecting energy consumption, geometry and materiality of an architectural arrangement;

- research on the development of complex energy consumption model to rapidly exchange information between geometrical form and thermal performance forecasting;

- development of sophisticated hybrid evolutionary optimization algorithms in architectural design software coupled with sophisticated and/or reduced heat transfer models for multiscale building applications. 
Finally, selected examples of evolutionary computational optimization models have been presented and discussed explicitly, while the main conclusions of past literature on the subject have been cited in detail. Additionally the main aspects that have been emphasized in several previous studies and must be considered in every attempt that aims to couple computational morphogenesis with interactive energy consumption optimization are resumed on the basis of a synthesis of past case studies findings. Hence, the main aim of the present review paper was to introduce the notion of computational morphogenesis in the building physics audience presenting at the same time the dominating optimization methods currently used on both scientific domains with the perspective to clearly present the guidelines for further development of this innovative and original research field. Withal, we observed that research on hybrid optimization methods especially developed for computational morphogenesis with the aim to improve heat storage and load shifting is limited. The main objective of this review paper was to revisit existing hybrid optimization techniques that can be developed (or coupled each other) with the objective of reducing the gap between architectural design and current scientific knowledge to optimize the building form through computational morphogenesis in early design stage, reducing its energy consumption. Thus, as we understand the main future challenge in computational morphogenesis remains to couple classic morphogenesis problems to classic thermal storage and load shifting problems in order to regenerate the iconic dimension of contemporary architecture in the framework of "climate change" reducing the energy impact of the building sector.

\section{Aknowledgements}

I would like to gratefully and sincerely thank the colleagues and great scholars Pascal Maillard, Jacques Haiesch, and Michaël Gutnic for their guidance, optimism, support, understanding, 
patience, and most importantly, their friendship during the recent difficult period when the present work has been realized. Furthermore the two anonymous reviewers are acknowledged here for their very constructive remarks and comments.

\section{References}

[1] S. Mahdi Ziaei, "Effects of financial development indicators on energy consumption and $\mathrm{CO} 2$ emission of European, East Asian and Oceania countries," Renew. Sustain. Energy Rev., vol. 42, pp. 752-759, Feb. 2015.

[2] E. Seinre, J. Kurnitski, and H. Voll, "Quantification of environmental and economic impacts for main categories of building labeling schemes," Energy Build., vol. 70, pp. 145-158, Feb. 2014.

[3] E. Pikas, J. Kurnitski, R. Liias, and M. Thalfeldt, "Quantification of economic benefits of renovation of apartment buildings as a basis for cost optimal 2030 energy efficiency strategies," Energy Build., vol. 86, pp. 151-160, Jan. 2015.

[4] J. Kurnitski, K. Kuusk, T. Tark, A. Uutar, T. Kalamees, and E. Pikas, "Energy and investment intensity of integrated renovation and 2030 cost optimal savings," Energy Build., vol. 75, pp. 51-59, Jun. 2014.

[5] N. Lepkova, D. Zubka, and R. L. Jensen, "Financial Investments for Zero Energy Houses," in Global Sustainable Communities Handbook, Elsevier, 2014, pp. 217-253.

[6] E. Pikas, M. Thalfeldt, and J. Kurnitski, “Cost optimal and nearly zero energy building solutions for office buildings," Energy Build., vol. 74, pp. 30-42, May 2014. 
[7] M. Thalfeldt, E. Pikas, J. Kurnitski, and H. Voll, "Facade design principles for nearly zero energy buildings in a cold climate," Energy Build., vol. 67, pp. 309-321, Dec. 2013.

[8] J. S. Bourrelle, "Zero energy buildings and the rebound effect: A solution to the paradox of energy efficiency?," Energy Build., vol. 84, pp. 633-640, Dec. 2014.

[9] L. Aelenei and H. Gonçalves, "From Solar Building Design to Net Zero Energy Buildings: Performance Insights of an Office Building,” Energy Procedia, vol. 48, pp. 1236-1243, 2014.

[10] Z. Szalay and A. Zöld, "Definition of nearly zero-energy building requirements based on a large building sample," Energy Policy, vol. 74, pp. 510-521, Nov. 2014.

[11] U. Desideri, L. Arcioni, D. Leonardi, L. Cesaretti, P. Perugini, E. Agabitini, and N. Evangelisti, “Design of a multipurpose 'zero energy consumption' building according to European Directive 2010/31/EU: Life cycle assessment," Energy Build., vol. 80, pp. 585597, Sep. 2014.

[12] S. Deng, R. Z. Wang, and Y. J. Dai, "How to evaluate performance of net zero energy building - A literature research,” Energy, vol. 71, pp. 1-16, Jul. 2014.

[13] M. Weibenberger, W. Jensch, and W. Lang, "The convergence of life cycle assessment and nearly zero-energy buildings: The case of Germany," Energy Build., vol. 76, pp. 551557, Jun. 2014.

[14] M. Ferrara, E. Fabrizio, J. Virgone, and M. Filippi, “A simulation-based optimization method for cost-optimal analysis of nearly Zero Energy Buildings,” Energy Build., vol. 84, pp. 442-457, Dec. 2014.

[15] W. Zeiler, "Dutch Efforts Towards a Sustainable Built Environment," in Sustainability, Energy and Architecture, Elsevier, 2013, pp. 1-25. 
[16] G. Salvalai, G. Masera, and M. M. Sesana, "Italian local codes for energy efficiency of buildings: Theoretical definition and experimental application to a residential case study," Renew. Sustain. Energy Rev., vol. 42, pp. 1245-1259, Feb. 2015.

[17] Y. Sun, "Sensitivity analysis of macro-parameters in the system design of net zero energy building," Energy Build., vol. 86, pp. 464-477, Jan. 2015.

[18] Y. Song, J. Sun, J. Li, and D. Xie, “Towards Net Zero Energy Building: Collaborationbased Sustainable Design and Practice of the Beijing Waterfowl Pavilion," Energy Procedia, vol. 57, pp. 1773-1782, 2014.

[19] F. Noris, E. Musall, J. Salom, B. Berggren, S. Ø. Jensen, K. Lindberg, and I. Sartori, "Implications of weighting factors on technology preference in net zero energy buildings," Energy Build., vol. 82, pp. 250-262, Oct. 2014.

[20] A. Mohamed, S. Cao, A. Hasan, and K. Sirén, "Selection of micro-cogeneration for net zero energy buildings (NZEB) using weighted energy matching index," Energy Build., vol. 80, pp. 490-503, Sep. 2014.

[21] I. Visa, M. D. Moldovan, M. Comsit, and A. Duta, "Improving the renewable energy mix in a building toward the nearly zero energy status," Energy Build., vol. 68, pp. 72-78, Jan. 2014.

[22] T. A.L. Martins, L. Adolphe, and L. E.G. Bastos, "From solar constraints to urban design opportunities: Optimization of built form typologies in a Brazilian tropical city," Energy Build., vol. 76, pp. 43-56, Jun. 2014.

[23] S. Papantoniou, D. Kolokotsa, and K. Kalaitzakis, "Building Optimization and Control algorithms implemented in existing BEMS using a Web based Energy Management and Control System," Energy Build., Nov. 2014. 
[24] M. D. Moldovan, I. Visa, M. Neagoe, and B. G. Burduhos, "Solar Heating \&amp; Cooling Energy Mixes to Transform Low Energy Buildings in Nearly Zero Energy Buildings,” Energy Procedia, vol. 48, pp. 924-937, 2014.

[25] S. Attia, M. Hamdy, W. O’Brien, and S. Carlucci, “Assessing gaps and needs for integrating building performance optimization tools in net zero energy buildings design," Energy Build., vol. 60, pp. 110-124, May 2013.

[26] X. Li and J. Wen, "Building energy consumption on-line forecasting using physics based system identification," Energy Build., vol. 82, pp. 1-12, Oct. 2014.

[27] L. E. Mavromatidis, A. Bykalyuk, M. El Mankibi, P. Michel, and M. Santamouris, 'Numerical estimation of air gaps' influence on the insulating performance of multilayer thermal insulation,” Build. Environ., vol. 49, pp. 227-237, Mar. 2012.

[28] A. Bykalyuk, F. Kuznik, and K. Johannes, "Studying the evolution of both thermal and kinetic boundary layers in the vicinity of a vertical conductive gypsum plate under dynamic time-depending conditions at the building scale," Energy Build., vol. 86, pp. 898-908, Jan. 2015.

[29] J. Vesterstrom and R. Thomsen, "A comparative study of differential evolution, particle swarm optimization, and evolutionary algorithms on numerical benchmark problems," 2004, pp. 1980-1987.

[30] C. Turhan, T. Kazanasmaz, I. E. Uygun, K. E. Ekmen, and G. G. Akkurt, "Comparative study of a building energy performance software (KEP-IYTE-ESS) and ANN-based building heat load estimation," Energy Build., vol. 85, pp. 115-125, Dec. 2014. 
[31] B. Belhadj, M. Bederina, Z. Makhloufi, A. Goullieux, and M. Quéneudec, "Study of the thermal performances of an exterior wall of barley straw sand concrete in an arid environment," Energy Build., vol. 87, pp. 166-175, Jan. 2015.

[32] L. E. Mavromatidis, A. Bykalyuk, and H. Lequay, "Development of polynomial regression models for composite dynamic envelopes' thermal performance forecasting," Appl. Energy, vol. 104, pp. 379-391, Apr. 2013.

[33] V. Machairas, A. Tsangrassoulis, and K. Axarli, “Algorithms for optimization of building design: A review," Renew. Sustain. Energy Rev., vol. 31, pp. 101-112, Mar. 2014.

[34] R. Evins, “A review of computational optimisation methods applied to sustainable building design," Renew. Sustain. Energy Rev., vol. 22, pp. 230-245, Jun. 2013.

[35] S. Roudavski, “Towards Morphogenesis in Architecture,” Int. J. Archit. Comput., vol. 7, no. 3, pp. 345-374, Sep. 2009.

[36] G. L. Baldo, G. Cesarei, S. Minestrini, and L. Sordi, "The EU Ecolabel scheme and its application to construction and building materials," in Eco-Efficient Construction and Building Materials, Elsevier, 2014, pp. 98-124.

[37] P. Tatsidjodoung, N. Le Pierrès, and L. Luo, “A review of potential materials for thermal energy storage in building applications," Renew. Sustain. Energy Rev., vol. 18, pp. 327-349, Feb. 2013.

[38] S. Doroudiani, B. Doroudiani, and Z. Doroudiani, "Materials that release toxic fumes during fire," in Toxicity of Building Materials, Elsevier, 2012, pp. 241-282.

[39] J. Temuujin, A. Minjigmaa, B. Davaabal, U. Bayarzul, A. Ankhtuya, T. Jadambaa, and K. J. D. MacKenzie, "Utilization of radioactive high-calcium Mongolian flyash for the 
preparation of alkali-activated geopolymers for safe use as construction materials," Ceram. Int., vol. 40, no. 10, pp. 16475-16483, Dec. 2014.

[40] C.-H. Young, Y.-L. Chen, and P.-C. Chen, "Heat insulation solar glass and application on energy efficiency buildings," Energy Build., vol. 78, pp. 66-78, Aug. 2014.

[41] M. Y. Shoeib and K. M. Thabayneh, "Assessment of natural radiation exposure and radon exhalation rate in various samples of Egyptian building materials," J. Radiat. Res. Appl. Sci., vol. 7, no. 2, pp. 174-181, Apr. 2014.

[42] L. E. Mavromatidis, M. El Mankibi, P. Michel, A. Bykalyuk, and M. Santamouris, "Guidelines to study numerically and experimentally reflective insulation systems as applied to buildings," Adv. Build. Energy Res., vol. 6, no. 1, pp. 2-35, May 2012.

[43] A.-T. Nguyen, S. Reiter, and P. Rigo, "A review on simulation-based optimization methods applied to building performance analysis," Appl. Energy, vol. 113, pp. 1043-1058, Jan. 2014.

[44] C. Lamnatou, D. Chemisana, R. Mateus, M. G. Almeida, and S. M. Silva, "Review and perspectives on Life Cycle Analysis of solar technologies with emphasis on buildingintegrated solar thermal systems," Renew. Energy, vol. 75, pp. 833-846, Mar. 2015.

[45] W.-H. Tsai, S.-J. Lin, J.-Y. Liu, W.-R. Lin, and K.-C. Lee, "Incorporating life cycle assessments into building project decision-making: An energy consumption and $\mathrm{CO} 2$ emission perspective," Energy, vol. 36, no. 5, pp. 3022-3029, May 2011.

[46] G. Han, J. Srebric, and E. Enache-Pommer, "Variability of optimal solutions for building components based on comprehensive life cycle cost analysis," Energy Build., vol. 79, pp. 223-231, Aug. 2014. 
[47] F. Stazi, A. Mastrucci, and P. Munafò, "Life cycle assessment approach for the optimization of sustainable building envelopes: An application on solar wall systems," Build. Environ., vol. 58, pp. 278-288, Dec. 2012.

[48] Y.-Y. Jing, H. Bai, and J.-J. Wang, "Multi-objective optimization design and operation strategy analysis of BCHP system based on life cycle assessment," Energy, vol. 37, no. 1, pp. 405-416, Jan. 2012.

[49] Y. Jin, L. Wang, Y. Xiong, H. Cai, Y. H. Li, and W. J. Zhang, "Feasibility studies on net zero energy building for climate considering: A case of 'All Green House' for Datong, Shanxi, China," Energy Build., vol. 85, pp. 155-164, Dec. 2014.

[50] E. Fabrizio, F. Seguro, and M. Filippi, "Integrated HVAC and DHW production systems for Zero Energy Buildings,” Renew. Sustain. Energy Rev., vol. 40, pp. 515-541, Dec. 2014.

[51] A. Gallo, B. T. Molina, M. Prodanovic, J. G. Aguilar, and M. Romero, "Analysis of Net Zero-energy Building in Spain. Integration of PV, Solar Domestic Hot Water and Airconditioning Systems," Energy Procedia, vol. 48, pp. 828-836, 2014.

[52] C. Yang, H. Li, Y. Rezgui, I. Petri, B. Yuce, B. Chen, and B. Jayan, "High throughput computing based distributed genetic algorithm for building energy consumption optimization," Energy Build., vol. 76, pp. 92-101, Jun. 2014.

[53] L. Junghans and N. Darde, "Hybrid single objective genetic algorithm coupled with the simulated annealing optimization method for building optimization," Energy Build., vol. 86, pp. 651-662, Jan. 2015.

[54] E. Asadi, M. G. da Silva, C. H. Antunes, L. Dias, and L. Glicksman, "Multi-objective optimization for building retrofit: A model using genetic algorithm and artificial neural network and an application," Energy Build., vol. 81, pp. 444-456, Oct. 2014. 
[55] S. Asadi, S. S. Amiri, and M. Mottahedi, "On the development of multi-linear regression analysis to assess energy consumption in the early stages of building design," Energy Build., vol. 85 , pp. 246-255, Dec. 2014.

[56] R. H. Myers, Response surface methodology: process and product optimization using designed experiments, 3rd ed. Hoboken, N.J: Wiley, 2009.

[57] B. Eisenhower, Z. O’Neill, S. Narayanan, V. A. Fonoberov, and I. Mezić, “A methodology for meta-model based optimization in building energy models," Energy Build., vol. 47, pp. 292-301, Apr. 2012.

[58] E. M. Malatji, J. Zhang, and X. Xia, "A multiple objective optimisation model for building energy efficiency investment decision," Energy Build., vol. 61, pp. 81-87, Jun. 2013.

[59] C. P. Quaglia, N. Yu, A. P. Thrall, and S. Paolucci, "Balancing energy efficiency and structural performance through multi-objective shape optimization: Case study of a rapidly deployable origami-inspired shelter,” Energy Build., vol. 82, pp. 733-745, Oct. 2014.

[60] D. E. Goldberg, Genetic algorithms in search, optimization, and machine learning. Reading, Mass: Addison-Wesley Pub. Co, 1989.

[61] V. Siddharth, P. V. Ramakrishna, T. Geetha, and A. Sivasubramaniam, “Automatic generation of energy conservation measures in buildings using genetic algorithms," Energy Build., vol. 43, no. 10, pp. 2718-2726, Oct. 2011.

[62] K. Li and H. Su, "Forecasting building energy consumption with hybrid genetic algorithm-hierarchical adaptive network-based fuzzy inference system," Energy Build., vol. 42, no. 11, pp. 2070-2076, Nov. 2010. 
[63] R. Ooka and K. Komamura, "Optimal design method for building energy systems using genetic algorithms," Build. Environ., vol. 44, no. 7, pp. 1538-1544, Jul. 2009.

[64] X. Xu and S. Wang, "Optimal simplified thermal models of building envelope based on frequency domain regression using genetic algorithm," Energy Build., vol. 39, no. 5, pp. 525-536, May 2007.

[65] J.-T. Jin and J.-W. Jeong, "Optimization of a free-form building shape to minimize external thermal load using genetic algorithm," Energy Build., vol. 85, pp. 473-482, Dec. 2014.

[66] J. A. Wright, H. A. Loosemore, and R. Farmani, “Optimization of building thermal design and control by multi-criterion genetic algorithm," Energy Build., vol. 34, no. 9, pp. 959-972, Oct. 2002.

[67] L. E. Mavromatidis, X. Marsault, and H. Lequay, "Daylight factor estimation at an early design stage to reduce buildings' energy consumption due to artificial lighting: A numerical approach based on Doehlert and Box-Behnken designs," Energy, vol. 65, pp. 488-502, Feb. 2014.

[68] Marsault, Xavier, "A multiobjective and interactive genetic algorithm to optimize the building form in early design stages," Build. Simul. 2013 Chambéry August 2013.

[69] Marin, Philipe, Marsault, Xavier, Mavromatidis, Lazaros Elias, Saleri, Renato, and Torres, Florent, "Ec-Co-Gen: An Evolutionary Simulation Assisted design Tool for Energy Rating of Buildings in Early Design Stage to Optimize the Building Form"," Build. Simul. 2013 Chambéry August 2013.

[70] C.-Y. Cui and B.-S. Jiang, “A morphogenesis method for shape optimization of framed structures subject to spatial constraints," Eng. Struct., vol. 77, pp. 109-118, Oct. 2014. 
[71] H. Yi, R. S. Srinivasan, and W. W. Braham, “An integrated energy-emergy approach to building form optimization: Use of EnergyPlus, emergy analysis and Taguchi-regression method," Build. Environ., vol. 84, pp. 89-104, Jan. 2015.

[72] M. Liu, M. Xing, Q. Yang, and X. Yang, "Computational morphogenesis of free form shell structures by optimization," Procedia Eng., vol. 31, pp. 608-612, 2012.

[73] S.-H. Lin and D. J. Gerber, "Evolutionary energy performance feedback for design: Multidisciplinary design optimization and performance boundaries for design decision support," Energy Build., vol. 84, pp. 426-441, Dec. 2014.

[74] C. Baglivo, P. M. Congedo, A. Fazio, and D. Laforgia, "Multi-objective optimization analysis for high efficiency external walls of zero energy buildings (ZEB) in the Mediterranean climate," Energy Build., vol. 84, pp. 483-492, Dec. 2014.

[75] M. Adamski, "Optimization of the form of a building on an oval base," Build. Environ., vol. 42, no. 4, pp. 1632-1643, Apr. 2007.

[76] J. H. Kämpf and D. Robinson, "Optimisation of building form for solar energy utilisation using constrained evolutionary algorithms," Energy Build., vol. 42, no. 6, pp. 807-814, Jun. 2010.

[77] Y. Yamaguchi, Y. Shimoda, and M. Mizuno, "Proposal of a modeling approach considering urban form for evaluation of city level energy management," Energy Build., vol. 39, no. 5, pp. 580-592, May 2007. 